\title{
EFFECTS OF ELASTICITY AND PRESSURE-SENSITIVE YIELDING ON PLANE-STRESS CRACK-TIP FIELDS
}

\author{
Z. E. A. BEN-AOUN and J. PAN \\ Department of Mechanical Engineering and Applied Mechanics, The University of Michigan, \\ Ann Arbor, MI 48109, U.S.A.
}

\begin{abstract}
The asymptotic plane-stress mode I crack-tip fields under small-scale yielding for pressuresensitive materials are investigated. The yield criterion for these materials is described by a linear combination of the effective stress and the hydrostatic stress. Plastic dilatancy is introduced by normality flow rule. A closed-form general asymptotic solution for singular centered fan sectors is given as a function of $\mu$, which is a pressure sensitivity parameter introduced in the yield condition. When elastic-perfectly plastic behavior is considered, the finite element results show the existence of elastic sectors bordering the stress-free crack faces. The near-tip stresses of the finite element results agree well with those of the corresponding asymptotic analysis. The angular spans of the elastic and plastic sectors vary with the value of $\mu$. The parameter $\mu$ also has significant effects on the sizes and shapes of the plastic zones. The contribution of hydrostatic stress in the yield criterion for this class of pressure-sensitive materials extends the boundary of the plastic zone much farther in front of the crack-tip than that for incompressible Mises materials.
\end{abstract}

\section{INTRODUCTION}

FOR ISOTROPIC materials, the yield condition can be expressed as a function of the three stress invariants; for example, see [1]. In the classical theory of plasticity, it is commonly agreed that the effects of the first invariant, which represents the hydrostatic stress, can be neglected. This was and still is a valid assumption for dense metals and alloys such as steels. Recently, there has been a great deal of research interest in toughened structural polymers and ceramics because of their outstanding mechanical properties. For both classes of materials, results of experiments suggest a constitutive description of yielding where the hydrostatic stress effect must be considered.

As discussed in [2], the effect of hydrostatic pressure on yield and plastic flow has been shown experimentally for a variety of metals and plastics. It could be inferred that hydrostatic pressure dependent yielding is one cause of the plastic volume increases of polymethyl methacrylate seen in the experiments by Whitney and Andrews [3]. Spitzig and Richmond [4] and Richmond and Spitzig [5] showed similar results for polyethylene and polycarbonate and concluded that hydrostatic stress has a significant influence on flow stress. More recently, Carapelluci and Yee [6] performed tests on glassy bisphenol-A polycarbonate and verified that a modified Mises criterion with linear dependence on hydrostatic stress gave a good fit for their experimental data. The phenomenon of pressure-sensitive yielding was also observed in tempered martensitic, maraging, and HY-80 steels by Spitzig et al. [7, 8]. However, the pressure sensitivity of yielding for steels is relatively low compared with those of polymers. Recently, similar pressure-sensitive yielding behavior was observed in transformation toughened ceramics by Chen and Reyes-Morel [9] and Reyes-Morel and Chen [10].

For isotropic pressure-insensitive materials the asymptotic crack-tip fields have been obtained by Hutchinson [11, 12], Rice and Rosengren [13], and Rice [14]. The asymptotic crack-tip fields for orthotropic materials can be found in [15-18]. For pressure-sensitive materials, both the asymptotic crack-tip power-law and perfect plastic solutions were given by $\mathrm{Li}$ and $\mathrm{Pan}[19,20]$. Note the radial stress discontinuity in plane-stress fully-yielded asymptotic crack-tip fields in [12,20]. Dong and Pan [21] carried out an elastic-plastic analysis of cracks under plane-strain and small-scale yielding for pressure-sensitive materials. Their elastic-perfectly plastic solutions show the existence of elastic sectors bordering the crack faces. The existence of elastic sectors, to eliminate the radial stress discontinuity, for elastic-perfectly plastic Mises material has also been verified by both finite element computations and asymptotic analysis in $[22,23]$ under mixed mode loading conditions.

In this paper, we study the effects of elasticity and pressure-sensitive yielding on mode I near-tip fields under plane-stress and small-scale yielding conditions. We first investigate 
the near-tip fields by finite element methods under monotonically increasing loading conditions until a steady stress state for the crack-tip elements is reached. Then, the asymptotic near-tip field is assembled and shown to be in full agreement with the finite element results.

\section{PRESSURE-SENSITIVE MATERIALS}

In this paper, we account for pressure-sensitive yielding in the yield condition by means of a linear combination of the hydrostatic stress $\sigma_{m}$ and the tensile effective stress $\sigma_{e}$ [2-10,24]. The yield condition can be written as

$$
\begin{aligned}
f(\boldsymbol{\sigma}) & =\sigma_{e}+\sqrt{3} \mu \sigma_{m}-\sigma_{0}=0 \\
\sigma_{e} & =\left(\frac{3}{2} s_{i j} s_{i j}\right)^{1 / 2} \quad s_{i j}=\sigma_{i j}-\sigma_{m} \delta_{i j} \quad \sigma_{m}=\sigma_{k k} / 3,
\end{aligned}
$$

where $s_{i j}$ are the deviatoric stresses, $\mu$ is a pressure sensitivity parameter, and $\sigma_{0}$ is a reference stress. This yield condition along with the familiar von Mises yield condition are plotted in Fig. 1.

We consider a plane crack problem as shown in Fig. 2 where the Cartesian coordinates $x_{1}$ and $x_{2}$ represent the in-plane coordinates and $x_{3}$ represents the out-of-plane coordinate. Under plane stress conditions, we have $\sigma_{13}=\sigma_{23}=\sigma_{33}=0$. Therefore, the yield function defined in eq. (1) can be expressed in the Cartesian coordinates as

$$
f(\sigma)=\left(\sigma_{11}^{2}+\sigma_{22}^{2}-\sigma_{11} \sigma_{22}+3 \sigma_{12}^{2}\right)^{1 / 2}+\frac{\mu}{\sqrt{3}}\left(\sigma_{11}+\sigma_{22}\right)-\sigma_{0}=0
$$

or in the polar coordinates, centered at the tip as shown in Fig. 2, as

$$
f(\sigma)=\left(\sigma_{r r}^{2}+\sigma_{\theta \theta}^{2}-\sigma_{r r} \sigma_{\theta \theta}+3 \sigma_{r \theta}^{2}\right)^{1 / 2}+\frac{\mu}{\sqrt{3}}\left(\sigma_{r r}+\sigma_{\theta \theta}\right)-\sigma_{0}=0 .
$$

Within the context of small-strain theory of plasticity, the total strain rate tensor can be decomposed into an elastic and a plastic part

$$
\dot{\epsilon}_{i j}=\dot{\epsilon}_{i j}^{e}+\dot{\epsilon}_{i j}^{p}
$$

The elastic strain rate tensor is given by

$$
\dot{\epsilon}_{i j}^{e}=M_{i j k l} \dot{\sigma}_{k l},
$$

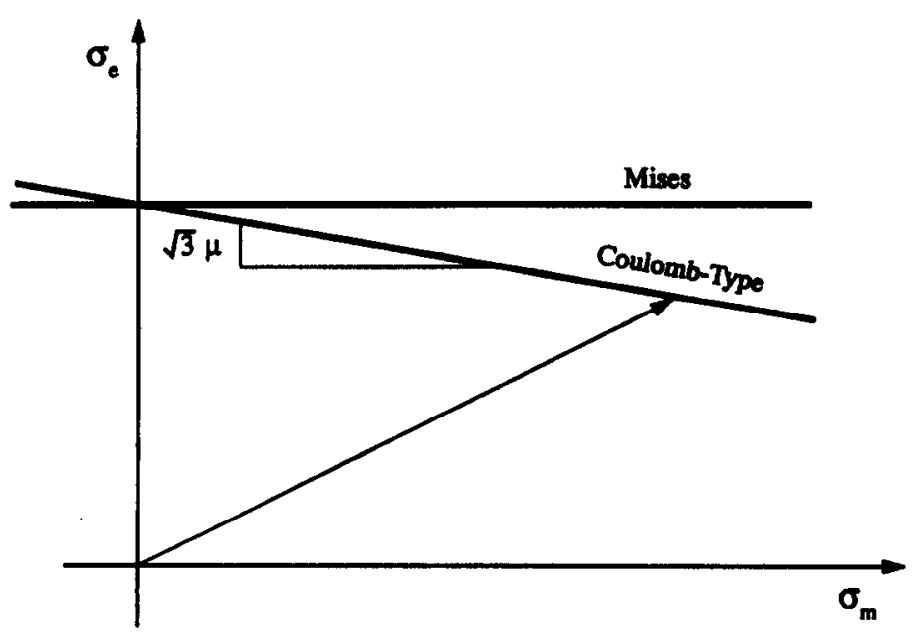

Fig. 1. Coulomb-type and Mises yield conditions. 


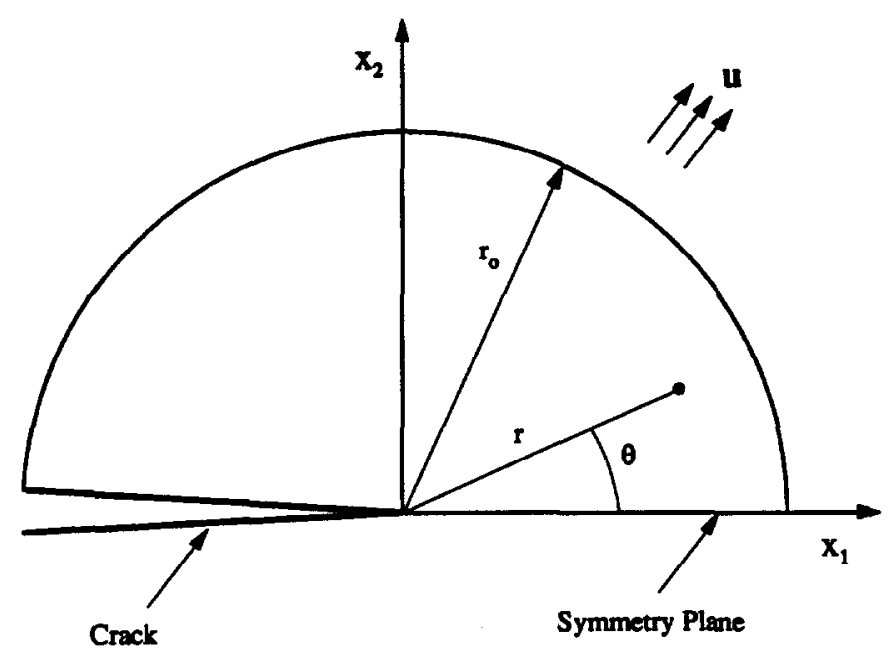

Fig. 2. Coordinate conventions and finite element model.

where $M_{i j k l}$ is a constant elastic positive definite compliance tensor. The plastic strain rate tensor is assumed to be normal to the yield surface and the flow rule is given by

$$
\dot{\epsilon}_{i j}^{p}=\lambda\left(\frac{3}{2} \frac{s_{i j}}{\sigma_{e}}+\frac{\mu}{\sqrt{3}} \delta_{i j}\right),
$$

where $\lambda$ is a positive proportionality factor. A detailed discussion on the pressure-sensitive constitutive relations can be found in $[25,26]$. Detailed information on a deformation plasticity version of the constitutive law can be found in [19].

\section{FINITE ELEMENT ANALYSIS}

\subsection{Computational model}

In this section, we will present a computational model for the small-scale yielding problem of a crack under mode I loading in an elastic-pressure-sensitive plastic material. We consider a crack in a circular domain of radius $r_{0}$. Due to the symmetry of mode I loading, we select the upper half of the circular domain as shown in Fig. 2, where $x_{1}$ and $x_{2}$ are the Cartesian coordinates, and $r$ and $\theta$ are the polar coordinates centered at the tip.

The interior area of the semi-circular domain is entirely discretized by a mesh of eight-node serendipity quadrilateral elements. The formulation and numerical integration as well as the advantages of this element have been widely discussed and can be found in detail in, for example, [27]. In the immediate crack-tip region, we use a ring of 18 wedge-shaped elements of size $r_{i}$ and we choose a ratio of $r_{i}$ to $r_{0}$ of $10^{-6}\left(r_{i} / r_{0}=10^{-6}\right)$. Elements in the $\theta$-direction are equally distributed from 0 to $\pi$. The wedge-shaped elements are surrounded by 24 semicircular strips generated by a logarithmic scale in the radial direction. Therefore, there is a total of 450 elements in the mesh. For the wedge-shaped elements, the collapsed node technique is employed to simulate the $1 / r$ singularity needed for the strain in the plastic sectors near the tip.

Along the remote circumferential boundary, the in-plane displacements are prescribed as

$$
u_{i}=\frac{K_{\mathrm{I}}}{2 G}\left(\frac{r}{2 \pi}\right)^{1 / 2} \tilde{u}_{i}^{\mathrm{I}}(\theta, v),
$$

where $G$ and $K_{1}$ represent the shear modulus and the far-field mode I stress intensity factor, respectively. The dimensionless functions $\tilde{u}_{i}^{1}(\theta, v)$ represent the asymptotic crack-tip displacements as functions of $\theta$ and Poisson's ratio $v$ for linear elastic materials (for example, see [28]). 


\subsection{Numerical procedure}

A general material nonlinear finite element analysis is performed effectively by use of an incremental formulation of the field equations. The finite element equations were derived from the principle of virtual work. At a time $t+\Delta t$ this takes the form

$$
\int_{A} \sigma_{t+\Delta t} \delta \varepsilon \mathrm{d} A=\int_{\partial A} \mathbf{T}_{t+\Delta t} \delta \mathbf{U d} S,
$$

where $\sigma_{t+\Delta t}$ represents the Cauchy stress tensor which satisfies the equilibrium at time $t+\Delta t, \mathbf{T}_{t+\Delta t}$ is the imposed traction vector on the boundary $\partial A, \delta \mathbf{U}$ represents the virtual displacement vector, and $\delta \varepsilon$ is the associated virtual small-strain tensor.

After linearizing eq. (8) about the equilibrium configuration at time $t$ and introducing the finite element approximation, the incremental equilibrium equations are obtained (see e.g. [29]). We specialize these equations to be solved by an iterative procedure based on a modified NewtonRaphson method. In matrix form, they are

$$
\mathbf{K}^{(i)} \Delta \mathbf{U}^{(i)}=\mathbf{R}_{i+\Delta t}-\mathbf{F}_{i+\Delta t}^{(i-1)}
$$

where $\mathbf{K}^{(j)}$ is the tangent stiffness matrix that corresponds to one of the previous equilibrium iterations $(1 \leqslant j \leqslant i-1)$; where $\Delta \mathbf{U}^{(i)}$ is the vector of the nodal point displacement increment at iteration $(i)$; where $\mathbf{R}_{t+\Delta t}$ is the vector of the externally applied nodal point loads which correspond to time $t+\Delta t$; and where $\mathbf{F}_{t+\Delta t}^{(i-1)}$ is the vector of the nodal point forces corresponding to the internal element stresses at time $t+\Delta t$ and iteration $(i-1)$ (in the first iteration, $i=1$, we use $F_{t}$ of the previous converged state). Note here that, for convenience, the discrete time increment $\Delta t$ denotes a load increment. The choice of iterations at which the stiffness matrix would be updated depends on the problem under consideration. Without any a priori knowledge of the system behavior, it may be efficient to update the stiffness matrix at every iteration for the first run $(j=i-1)$.

We first initiate the loading by a small value so that all the elements remain elastic. Then, since we are still in the elastic region, we can scale the load to cause yielding at the integration point of the element with the highest generalized effective stress $\sigma_{g e}\left(=\sigma_{e}+\sqrt{3} \mu \sigma_{m}\right)$, which belongs to, in our case, the wedge-shaped element right in front of the crack. This load for which we obtain the initial yielding is called the critical load. From this stage, the load is incremented with a small fraction of the critical load.

For each increment of load, the iterative procedure, described above, is used until the equilibrium [eq. (9)] is satisfied. We measure the convergence of the incremental equilibrium equation by the following ratio:

$$
\frac{\left\|\mathbf{R}_{t+\Delta t}-\mathbf{F}_{t+\Delta t}^{(i-1)}\right\|}{\left\|\mathbf{R}_{t+\Delta t}\right\|} \leqslant \delta_{F},
$$

where $\|\cdot\|$ is the Euclidean norm and $\delta_{F}$ is the tolerance that we wish to obtain in our calculation.

When any iterative algorithm is applied to a history dependent problem, the intermediate nonconverged solutions obtained during the iteration processes are usually not on the actual solution path, and thus the history dependent variables must be performed at the end of the converged iteration for each load increment, and not obtained as the sum of integrations associated with each iteration. In our program, this is done by integrating the field variable from the previously converged state. Then, we need to apply this procedure to the stress field so that the stresses at any iteration $(i)$ are computed from the values of the last converged equilibrium state as follows:

$$
\sigma_{i+\Delta t}^{(i)}=\sigma_{t}+\int_{\delta_{r}}^{d_{i}^{(i)}+\Delta t} \mathrm{D}_{e p} \mathrm{~d} \boldsymbol{\varepsilon},
$$

where $\mathbf{D}_{e p}$ is the elastic-plastic material constitutive matrix. This eliminates any error accumulation during intermediate iterations (see $[22,30]$ ).

\subsection{Numerical results}

For engineering materials, the values of $\mu$ are usually about $0.02-0.06$ for steels (see e.g. [5]), $0.1-0.25$ for polymers (see [31]), and $0.55-0.77$ for transformation toughened ceramics (see $[9,10]$ ). 
Therefore, the computations were performed for three values of pressure sensitivity, $\mu=0$ (for pressure-insensitive Mises material), 0.4 , and 0.8 . In all the results reported in the following, the ratio of Young's modulus $E$ to the tensile yield stress $\sigma_{0}$ was taken as 500 , and Poisson's ratio $v$ was taken as 0.3 .

The crack-tip stress fields are shown in Fig. 3. In these plots, the symbols represent the stresses of the Gauss points which form a fan surrounding the crack-tip at a distance defined by $r / r_{p} \approx 10^{-2}$ (here $r_{p}$ represents the plastic zone size ahead of the tip at $\theta=0$ ). The distance is defined in this manner to ensure that these Gauss points in plastic sectors are well inside the plastic zone but on the other hand outside the crack-tip region where the finite deformation effects dominate. The solid lines in Fig. 3 represent the stresses evaluated by the corresponding asymptotic analysis which will be detailed in Section 4. In these figures, $\sigma_{g e}$ represents the generalized effective stress which is equal
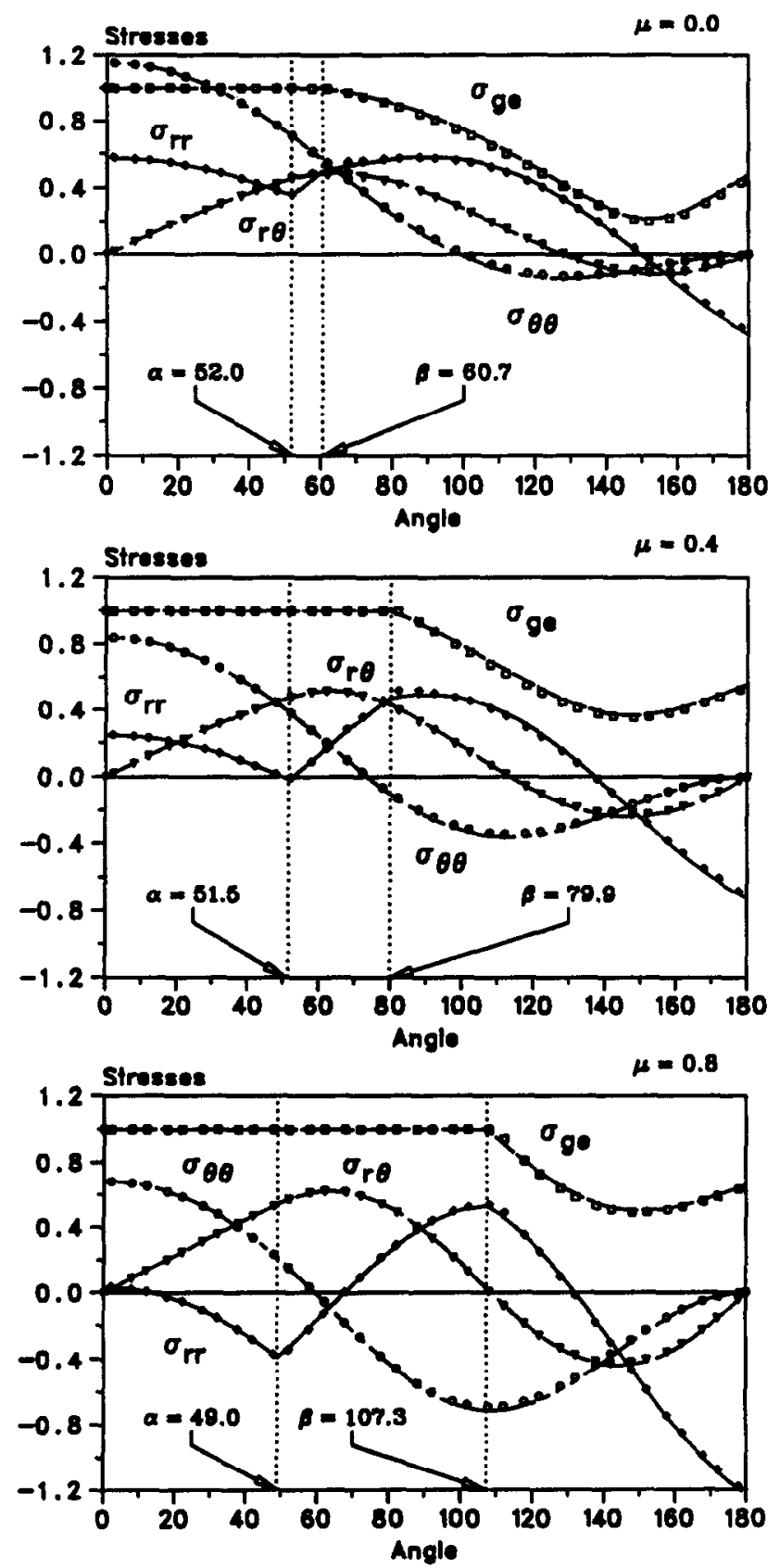

Fig. 3. Angular stress distributions for elastic-perfectly plastic materials with various pressure sensitivity parameters. Top: $\boldsymbol{\mu}=\mathbf{0}$. Center: $\mu=0.4$. Bottom: $\mu=0.8$. 
to $\sigma_{c}+\sqrt{3} \mu \sigma_{m}$, and all the stresses are normalized by the reference stress $\sigma_{0}$. It is important to note that all the components of the stresses, including $\sigma_{r r}$, are continuous, in contrast to the asymptotic solutions of Hutchinson [12] and Dong and Pan [23] for Mises materials, and Li and Pan [20] for pressure-sensitive materials, where the discontinuity of $\sigma_{r r}$ appears between two constant stress sectors because of the assumption that the material surrounding the crack-tip is fully yielded.

\section{ASYMPTOTIC CRACK-TIP FIELDS}

\subsection{Singular plastic sectors}

The outline of the analysis in this section is based on the work by Rice [32]. The analysis is valid for an arbitrary anisotropy of homogeneous materials. Its only restriction is the requirement of smooth yield surfaces.

As $r \rightarrow 0$, for plane stress problems $\left(\sigma_{13}=\sigma_{23}=\sigma_{33}=0\right)$, the equilibrium equations given by Rice [14] are

$$
\begin{gathered}
\sigma_{r r}-\sigma_{\theta \theta}+\sigma_{r \theta}^{\prime}=0 \\
2 \sigma_{r \theta}+\sigma_{\theta \theta}^{\prime}=0,
\end{gathered}
$$

where "" means differentiation with respect to $\theta$. Combining the differential form of the yield condition with the equilibrium equations gives

$$
\left(\sigma_{11}^{\prime}+\sigma_{22}^{\prime}\right) P_{r r}=0
$$

where

$$
P_{r r}=\frac{\partial f(\sigma)}{\partial \sigma_{r r}}
$$

There are three equations [eqs (12), (13), and (14)] for three unknown stresses. Therefore, this is a statically determinate problem. From eq. (14)

$$
\text { either } P_{r r}=0 \text { or } \sigma_{11}^{\prime}+\sigma_{22}^{\prime}=0
$$

must be true. Let us discuss both cases in the following.

4.1.1. Centered fan sector. $P_{r r}=0$ defines a fan of radial characteristics as follows:

$$
P_{r r}=\frac{\partial f(\sigma)}{\partial \sigma_{r r}}=0
$$

or

$$
\frac{1}{2}\left(2 \sigma_{r r}-\sigma_{\theta \theta}\right)+\frac{\mu}{\sqrt{3}} \sigma_{e}=0
$$

Using eq. (16) and the equilibrium equations [eqs (12) and (13)] as well as the yield criterion [eq. (3)], we can solve for the three stress components. We are interested in solutions for $\mu \leqslant 0.8$. However, two similarly structured solutions arise, depending on the sign of the discriminant of the characteristic equation for $0 \leqslant \mu<\sqrt{3}$. The first case for $0 \leqslant \mu<\sqrt{3} / 2$ is of periodic nature:

$$
\begin{gathered}
\sigma_{\theta \theta}=F_{1} \cos \rho\left(\theta-\theta_{0}\right)+F_{2} \sin \rho\left(\theta-\theta_{0}\right)-\frac{2 \sqrt{3} \mu}{3-4 \mu^{2}} \sigma_{0} \\
\sigma_{r r}=\frac{3+2 \mu^{2}}{2\left(3-\mu^{2}\right)}\left[F_{1} \cos \rho\left(\theta-\theta_{0}\right)+F_{2} \sin \rho\left(\theta-\theta_{0}\right)\right]-\frac{2 \sqrt{3} \mu}{3-4 \mu^{2}} \sigma_{0} \\
\sigma_{r \theta}=\frac{\rho F_{1}}{2} \sin \rho\left(\theta-\theta_{0}\right)-\frac{\rho F_{2}}{2} \cos \rho\left(\theta-\theta_{0}\right),
\end{gathered}
$$

where $\rho^{2}=\left(3-4 \mu^{2}\right) /\left(3-\mu^{2}\right)$. Here $\theta_{0}$ represents a reference angle. The constants $F_{1}$ and $F_{2}$ are related by

$$
F_{1}^{2}+F_{2}^{2}=4 \frac{3-\mu^{2}}{\left(3-4 \mu^{2}\right)^{2}} \sigma_{0}^{2}
$$


When $\mu=\sqrt{3} / 2$, the stress field is quadratic in nature:

$$
\begin{gathered}
\sigma_{\theta \theta}=-\frac{2}{3} \sigma_{0}\left(\theta-\theta_{0}\right)^{2}-2 F_{3}\left(\theta-\theta_{0}\right)+F_{4} \\
\sigma_{r r}=-\frac{2}{3} \sigma_{0}\left(\theta-\theta_{0}\right)^{2}-2 F_{3}\left(\theta-\theta_{0}\right)+F_{4}-\frac{2}{3} \sigma_{0} \\
\sigma_{r \theta}=\frac{2}{3} \sigma_{0}\left(\theta-\theta_{0}\right)+F_{3},
\end{gathered}
$$

where $F_{3}$ and $F_{4}$ are related by

$$
F_{3}^{2}+\frac{2}{3} F_{4} \sigma_{0}=\frac{4}{9} \sigma_{0}^{2} .
$$

The second case for $\sqrt{3} / 2<\mu<\sqrt{3}$ is aperiodic:

$$
\begin{gathered}
\sigma_{\theta \theta}=F_{5} \cosh \bar{\rho}\left(\theta-\theta_{0}\right)+F_{6} \sinh \bar{\rho}\left(\theta-\theta_{0}\right)-\frac{2 \sqrt{3} \mu}{3-4 \mu^{2}} \sigma_{0} \\
\sigma_{r r}=\frac{3+2 \mu^{2}}{2\left(3-\mu^{2}\right)}\left(F_{5} \cosh \bar{\rho}\left(\theta-\theta_{0}\right)+F_{6} \sinh \bar{\rho}\left(\theta-\theta_{0}\right)\right)-\frac{2 \sqrt{3} \mu}{3-4 \mu^{2}} \sigma_{0} \\
\sigma_{r \theta}=-\frac{\bar{\rho} F_{5}}{2} \sinh \bar{\rho}\left(\theta-\theta_{0}\right)-\frac{\bar{\rho} F_{6}}{2} \cosh \bar{\rho}\left(\theta-\theta_{0}\right),
\end{gathered}
$$

where $\bar{\rho}^{2}=\left(3-4 \mu^{2}\right) /\left(3-\mu^{2}\right)$. The constants $F_{5}$ and $F_{6}$ have to satisfy a condition similar to the periodic case, namely

$$
F_{5}^{2}-F_{6}^{2}=4 \frac{3-\mu^{2}}{\left(3-4 \mu^{2}\right)^{2}} \sigma_{0}^{2} .
$$

Note that $F_{1}, F_{2}, F_{3}, F_{4}, F_{5}$, and $F_{6}$ are constants which will be determined by the boundary conditions.

The Mises material is a special case of the first set of solutions with $\mu=0$ and $\rho^{2}=1$. For this case the stress field becomes

$$
\begin{gathered}
\sigma_{\theta \theta}=F_{1} \cos \left(\theta-\theta_{0}\right)+F_{2} \sin \left(\theta-\theta_{0}\right) \\
\sigma_{r r}=\frac{1}{2}\left(F_{1} \cos \left(\theta-\theta_{0}\right)+F_{2} \sin \left(\theta-\theta_{0}\right)\right)=\frac{1}{2} \sigma_{\theta \theta} \\
\sigma_{r \theta}=\frac{1}{2}\left(F_{1} \sin \left(\theta-\theta_{0}\right)-F_{2} \cos \left(\theta-\theta_{0}\right)\right),
\end{gathered}
$$

where $F_{1}^{2}+F_{2}^{2}=\frac{4}{3} \sigma_{0}^{2}$. This is consistent with the slip-line solution for Mises materials under plane stress conditions.

4.1.2. Constant stress sector. $\sigma_{11}^{\prime}+\sigma_{22}^{\prime}=0$ leads to $\sigma_{11}, \sigma_{22}$, and $\sigma_{12}$ being constants. This represents a constant stress sector. These constants must be chosen to satisfy the yield function and the boundary conditions.

\subsection{Elastic sectors}

The analysis in this section is valid for any elastic isotropic materials under both plane strain and plane stress conditions. The compatibility equation for the elastic sectors of finite stresses is [22]

$$
\left(\sigma_{r r}+\sigma_{\theta \theta}\right)^{\prime \prime}=0
$$

where "'"” represents the second differentiation with respect to $\theta$. By integrating the above compatibility condition [eq. (32)], we obtain

$$
\sigma_{r r}+\sigma_{\theta \theta}=4 B \theta+2 A \text {. }
$$

Combining eq. (33) with the equilibrium equations gives [22]

$$
\begin{gathered}
\sigma_{r r}=A+2 B \theta-C \cos 2 \theta-D \sin 2 \theta \\
\sigma_{\theta \theta}=A+2 B \theta+C \cos 2 \theta+D \sin 2 \theta \\
\sigma_{r \theta}=-B+C \sin 2 \theta-D \cos 2 \theta,
\end{gathered}
$$

where $A, B, C$ and $D$ are constants. 


\subsection{Assembly of crack-tip solution}

In this paper, we only consider mode I symmetric cases, which lead us to the following familiar symmetry condition:

$$
\sigma_{r \theta}(\theta=0)=0
$$

The stress free boundary conditions are

$$
\sigma_{r \theta}(\theta=\pi)=\sigma_{\theta \theta}(\theta=\pi)=0
$$

4.3.1. Elastic-plastic solution. Based on our numerical results, the assembled asymptotic solutions should feature the following:

-continuity of the stress field, including $\sigma_{r r}$

- elastic sectors bordering the faces of the crack.

Guided by the perfect-plasticity asymptotic solution assembled by Hutchinson [12], by the elastic-plastic solution assembled by Dong and Pan [23], and by our numerical results, we clearly note the formation of three distinct sectors: a centered fan sector in front of the crack-tip bordered by a constant stress sector which is in turn bordered by an elastic sector leading to the traction free face of the crack. These sectors are plotted in a schematic form in Fig. 4, where C.F. denotes a centered fan sector and C.S. denotes a constant stress sector.
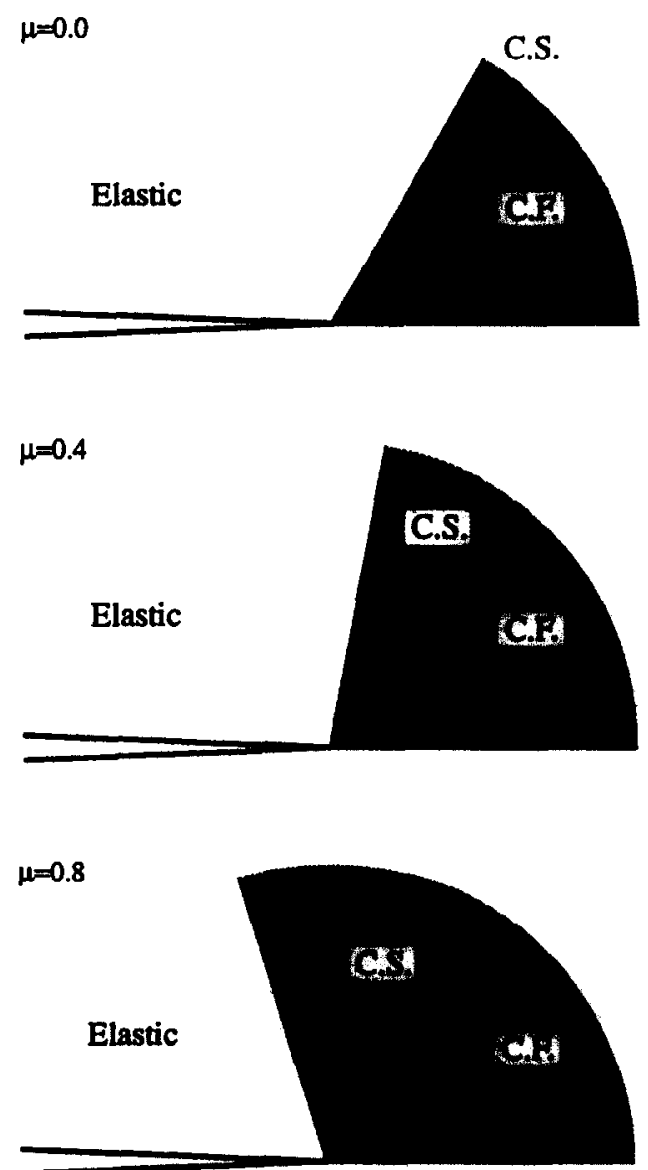

Fig. 4. Asymptotic crack-tip structures for elastic-perfectly plastic materials with various pressure sensitivity parameters. Top: $\mu=0$. Center: $\mu=0.4$. Bottom: $\mu=0.8$. 
In front of the crack-tip at $\theta=0$, we use the fact that our loading is symmetric [eq. (37)] and we take $\sigma_{r r}=c \sigma_{\theta \theta}$, where $c$ is a function of $\mu$. Using the latter assumption in the yield function [eq. (3)], we can solve for $\sigma_{\theta \theta}$ as follows (see [20]):

$$
\sigma_{\theta \theta}=\left[\left(c^{2}-c+1\right)^{1 / 2}+\frac{\mu}{\sqrt{3}}(c+1)\right]^{-1} \sigma_{0} .
$$

Now using eq. (16), we can solve for the proportionality constant $c$

$$
c=\frac{1}{2}\left(1 \pm \frac{\mu}{\left(1-\frac{\mu^{2}}{3}\right)^{1 / 2}}\right)
$$

Note that, based on the condition of eq. (16) and the fact that $\sigma_{e}$ is positive, $2 \sigma_{r r}-\sigma_{\theta \theta}$ has to be negative which implies that $c$ has to be less than $1 / 2$. Then, we should use only the negative sign in eq. (40). The formula for the constant $c$ becomes

$$
c=\frac{1}{2}\left(1-\frac{\mu}{\left(1-\frac{\mu^{2}}{3}\right)^{1 / 2}}\right) .
$$

Note that when $\mu=\sqrt{3} / 2, c=0$. This implies that the radial stress $\sigma_{r r}$ is 0 at $\theta=0$. When $\mu$ is in the second interval $(\sqrt{3} / 2<\mu<\sqrt{3}), c$ is less than zero. This indicates that $\sigma_{r r}$ is negative.

Using eq. (39), eq. (41), and the symmetry of the centered fan sector ahead of the tip which requires $\theta_{0}=0$ and $F_{2}=0$, we can solve for the constant $F_{1}$ in eqs (17)-(19). The stress field for the centered fan sector becomes

$$
\begin{gathered}
\sigma_{\theta \theta}=\frac{2}{\sqrt{3}} \frac{\sigma_{0}}{1-\frac{4}{3} \mu^{2}}\left(\sqrt{\left.\left(1-\frac{\mu^{2}}{3}\right) \cos \rho \theta-\mu\right)}\right. \\
\sigma_{r r}=\frac{2}{\sqrt{3}} \frac{\sigma_{0}}{1-\frac{4}{3} \mu^{2}}\left(\frac{1+\frac{2}{3} \mu^{2}}{2 \sqrt{\left(1-\frac{\mu^{2}}{3}\right)}} \cos \rho \theta-\mu\right) \\
\sigma_{r \theta}=\frac{1}{\sqrt{3}} \frac{\sigma_{0}}{\sqrt{ }\left(1-\frac{4}{3} \mu^{2}\right)} \sin \rho \theta .
\end{gathered}
$$

Again the stress field for Mises materials would be the limit of the above system when $\mu$ goes to zero. When $\mu=\sqrt{3} / 2$, the stress field for the centered fan in front of the tip is

$$
\begin{gathered}
\sigma_{\theta \theta}=-\frac{2}{3} \sigma_{0}\left(\theta^{2}-1\right) \\
\sigma_{r r}=-\frac{2}{3} \sigma_{0} \theta^{2} \\
\sigma_{r \theta}=\frac{2}{3} \sigma_{0} \theta^{2} .
\end{gathered}
$$

Note that we can also get the solutions for $\sqrt{3} / 2<\mu<\sqrt{3}$ by just replacing $\rho$ by $\bar{\rho}$ and the trigonometric functions by their hyperbolic counterparts in eqs (42)-(44).

For the elastic region, being extended to the face of the crack, we need to use the boundary conditions at $\theta=\pi$ [eq. (38)]. Applying these boundary conditions to eqs (34)-(36), the stress field in the elastic region becomes

$$
\begin{gathered}
\sigma_{r r}=A(1+\cos 2 \theta)+B(2 \theta+2 \pi \cos 2 \theta+\sin 2 \theta) \\
\sigma_{\theta \theta}=A(1-\cos 2 \theta)+B(2 \theta-2 \pi \cos 2 \theta-\sin 2 \theta) \\
\sigma_{r \theta}=-A \sin 2 \theta-B(1+2 \pi \sin 2 \theta-\cos 2 \theta) .
\end{gathered}
$$


We can express the stress field in the constant stress sector in terms of the angle $\alpha$ defined in Fig. 3:

$$
\begin{gathered}
\sigma_{11}=\sigma_{r r}(\alpha) \cos ^{2} \alpha+\sigma_{\theta \theta}(\alpha) \sin ^{2} \alpha-2 \sigma_{r \theta}(\alpha) \sin \alpha \cos \alpha \\
\sigma_{22}=\sigma_{r r}(\alpha) \sin ^{2} \alpha+\sigma_{\theta \theta}(\alpha) \cos ^{2} \alpha+2 \sigma_{r \theta}(\alpha) \sin \alpha \cos \alpha \\
\sigma_{12}=\left(\sigma_{r r}(\alpha)-\sigma_{\theta \theta}(\alpha)\right) \sin \alpha \cos \alpha+\sigma_{r \theta}(\alpha)\left(\cos ^{2} \alpha-\sin ^{2} \alpha\right),
\end{gathered}
$$

where $\sigma_{\theta \theta}(\alpha), \sigma_{r r}(\alpha)$, and $\sigma_{r \theta}(\alpha)$ are the stresses of the centered fan region evaluated at $\theta=\alpha$ in eqs (42)-(44).

We can solve for $\beta$, defined in Fig. 3, by assuming the continuity of $\sigma_{r \theta}$ and $\sigma_{\theta \theta}$ between the constant stress sector and the elastic sector and forcing the stress field of the elastic region to satisfy
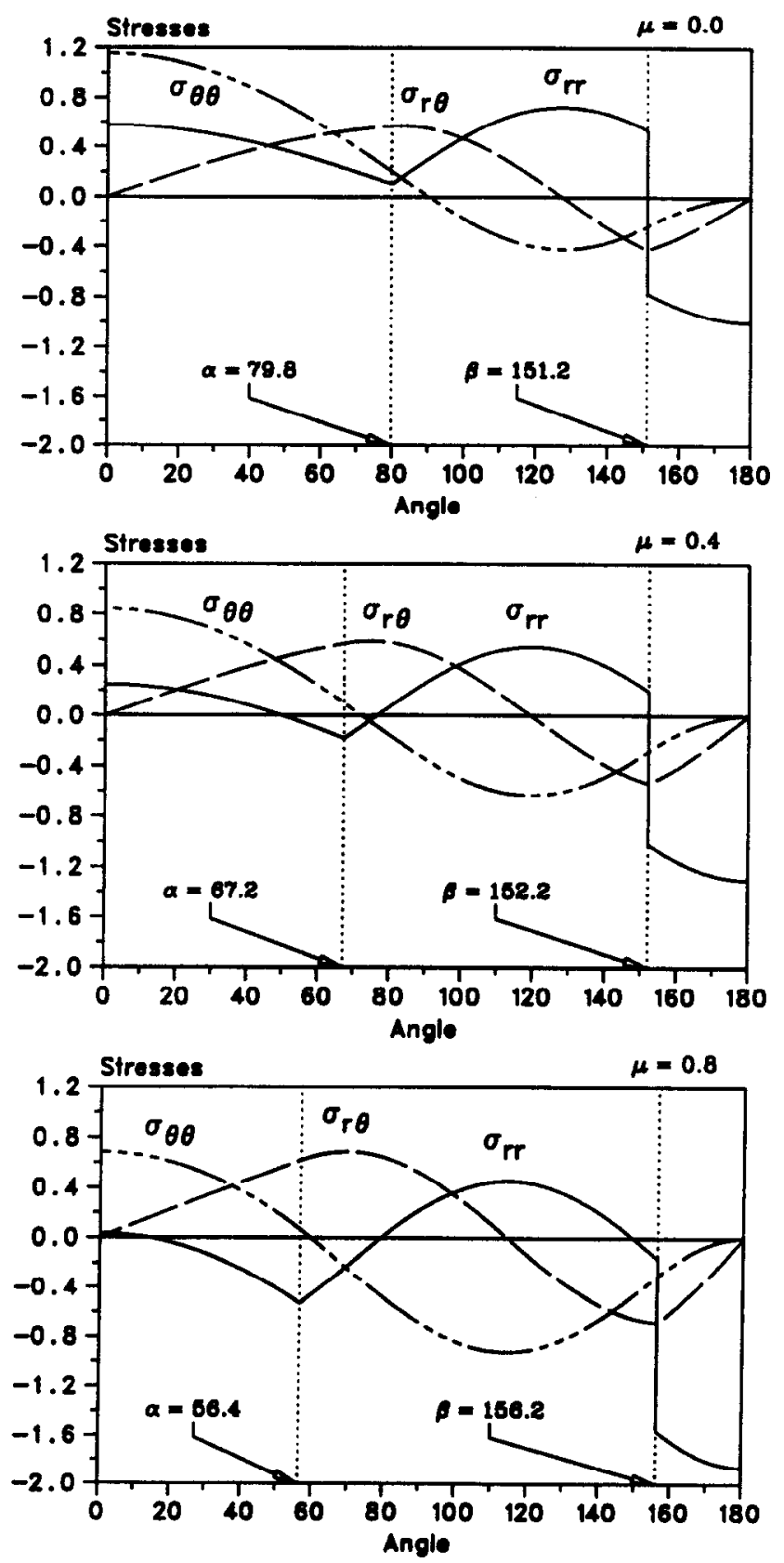

Fig. 5. Fully-yielded angular stress distributions for perfectly plastic materials with various pressure sensitivity parameters. Top: $\mu=0$. Center: $\mu=0.4$. Bottom: $\mu=0.8$. 
the yield function [eq. (3)] at $\theta=\beta$. Note that the continuity of $\sigma_{r r}$ is guaranteed because the yield function as well as the continuity of $\sigma_{r \theta}$ and $\sigma_{\theta \theta}$ are satisfied at $\theta=\beta$. These conditions form a system of three equations for the three unknowns $A, B$, and $\beta$. To solve it, we use a solution procedure based on Newton's method routine to satisfy the yield function. This results in a uniquely determined angle $\beta$ as well as a unique set of constants $A$ and $B$ as functions of $\alpha$ which is estimated from the finite element solutions. Thus, we can fully determine the stress field in all sectors. As expected, the asymptotic solution resulted in a continuous stress field throughout the region as shown by solid lines in Fig. 3. The asymptotic solutions agree well with the computational results shown as the symbols in Fig. 3.
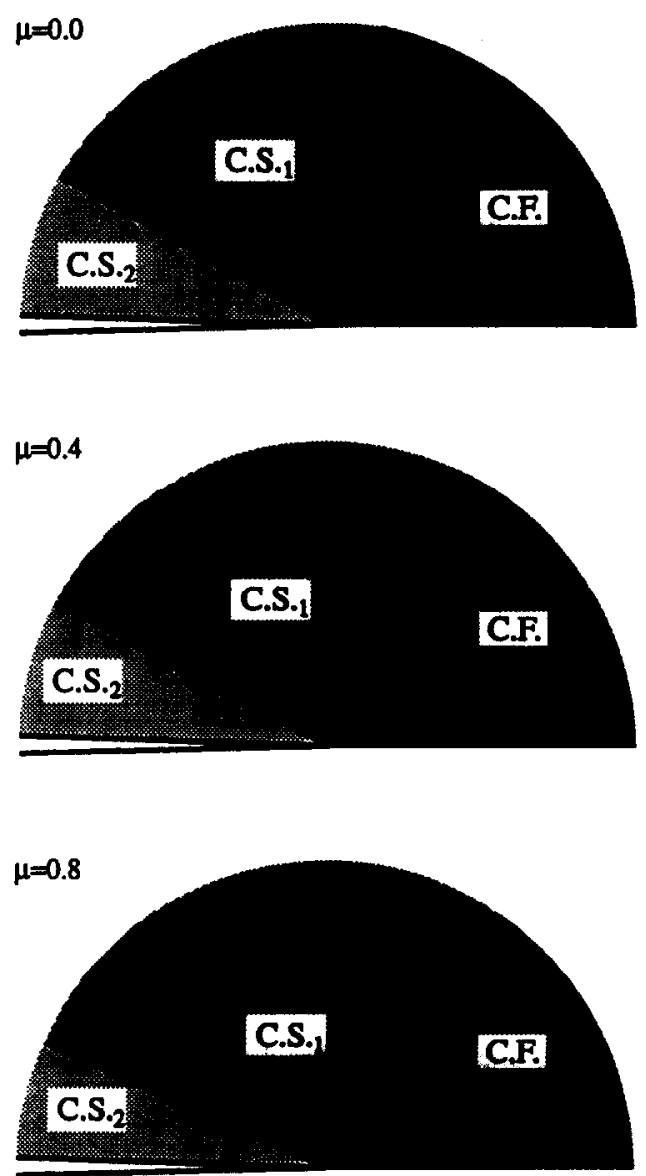

Fig. 6. Fully-yielded asymptotic crack-tip structures for perfectly plastic materials with various pressure sensitivity parameters. Top: $\mu=0$. Center: $\mu=0.4$. Bottom: $\mu=0.8$.
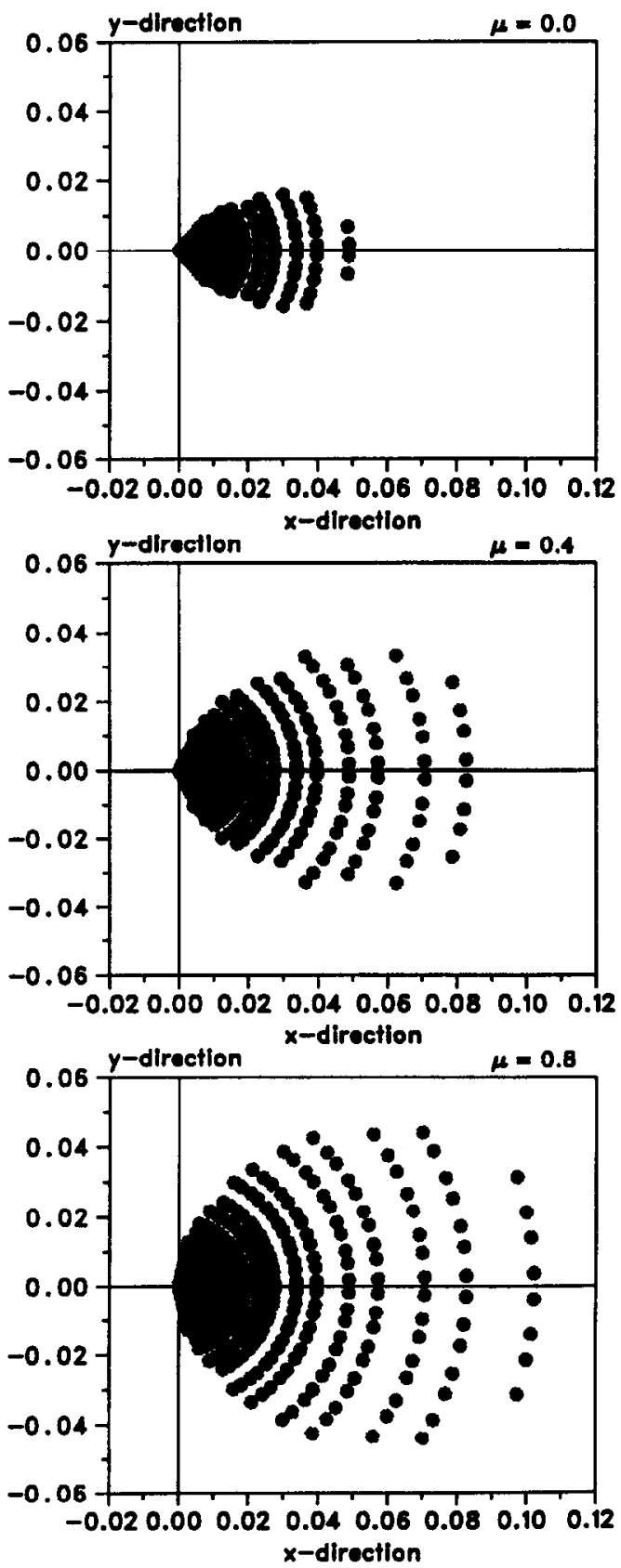

Fig. 7. Plastic zones normalized by $J E / \sigma_{0}^{2}$ for various pressure sensitivity parameters. Top: $\mu=0$. Center: $\mu=0.4$. Bottom: $\mu=0.8$ 
4.3.2. Fully-yielded perfectly plastic solutions. For comparison, we present the corresponding fully-yielded solutions for perfectly plastic materials. These plane-stress asymptotic solutions were given by $\mathrm{Li}$ and Pan [20]. The assembled crack-tip fields consist of a centered fan sector in front of the crack-tip and two adjacent constant stress sectors between the centered fan sector and the face of the crack. The stress fields normalized by the reference stress $\sigma_{0}$ for $\mu=0,0.4$, and 0.8 are plotted in Fig. 5 and the corresponding asymptotic crack-tip structures are shown in Fig. 6 (where C.F. denotes a centered fan sector, and C.S..$_{1}$ and C.S..$_{2}$ represent two constant stress sectors).

Although the fully-yielded solutions are not supported by our finite element results, they can still be a good case for comparison. The regions near which the fully-yielded solutions predict the discontinuities of $\sigma_{r r}$, as shown in Fig. 5 (note the increasing discontinuity as $\mu$ increases), are actually replaced by elastic regions and all the stresses are continuous as shown in the elastic-plastic solutions in Fig. 3. Note that, for each $\mu$, the stress states ahead of the tip at $\theta=0$ are the same for both elastic-plastic and fully plastic fields. However, the angular spans of the centered fan and constant stress sectors are different for both fields. On the other hand, we notice similar characteristics such as the decrease of the span of the centered fan region $\alpha$ as well as the increase of the span of the constant stress sector (bordering the centered fan sector) and the total yielded angular $\operatorname{span} \beta$, when $\mu$ increases. These features are shown in Figs 3 and 5 for the stress distributions as well as for the values of $\alpha$ and $\beta$ and in Figs 4 and 6 for the actual sector spans.

\section{DISCUSSION}

In this paper, we have studied the plane-stress crack-tip fields for the elastic-plastic pressure-sensitive materials. We have shown in our numerical studies the existence of elastic sectors bordering the face of the crack (see Figs 3 and 4). The issue of the existence of the elastic sector for elastic-perfectly plastic materials was raised by Gao [33], Nemat-Nasser and Obata [34], Saka et al. [35], Dong and Pan [22, 23] for Mises materials and Dong and Pan [21] for pressure-sensitive materials. In the analysis of the pure mode I plane-stress case, Dong and Pan [23] overlooked the existence of the constant stress sector that we have noticed clearly in our study especially at a large pressure-sensitivity parameter.

The plastic zones from the finite element solutions in steady state, normalized by $J E / \sigma_{0}^{2}$ (here $J=K_{1}^{2} / E$ ), are shown in Fig. 7 for $\mu=0,0.4$, and 0.8 . The dots in these plots represent Gauss points in their plastic state. In these figures, we can see the extension of the angular span of the plastic region as seen earlier in Figs 3 and 4. We also notice that the normalized plastic zone enlarges as $\mu$ increases. Also, as $\mu$ increases, the crack-tip stresses ahead of the crack decrease. This may partially explain the toughening effect due to an increase of macroscopic pressure sensitivity by introducing microvoids, microcracks, and phase transformation.

Acknowledgements-Z. E. A. Ben-Aoun acknowledges the support of the University Mission of Tunisia for its scholarship. The authors also acknowledge the support of this work by the National Science Foundation under grant numbers MSM-8613544 and DMR-8708405.

\section{REFERENCES}

[1] R. Hill, The Mathematical Theory of Plasticity. Oxford University Press, Oxford (1950).

[2] D. C. Drucker, Plasticity theory, strength-differential (SD) phenomenon, and volume expansion in metals and plastics. Metall. Trans. 4, 667-673 (1973).

[3] W. Whitney and R. D. Andrews, Yielding of glassy polymers: volume effects. J. Polymer Sci.: Polymer Symposia 16C, 2981-2990 (1967).

[4] W. A. Spitzig and O. Richmond, Effect of hydrostatic pressure on the deformation behavior of polyethylene and polycarbonate in tension and in compression. Polymer Engng Sci. 19, 1129-1139 (1979).

[5] O. Richmond and W. A. Spitzig, Pressure dependence and dilatancy of plastic flow. Int. Union theor. appl. Mech. $377-386$ (1980).

[6] L. M. Carapelluci and A. F. Yee, The biaxial deformation and yield behavior of bisphenol-A polycarbonate: effect of anisotropy. Polymer Engng Sci. 26, 920-930 (1986).

[7] W. A. Spitag, R. J. Sober and O. Richmond, Pressure dependence of yielding and associated volume expansion in tempered martensite. Acta Metall. 23, 885-893 (1975).

[8] W. A. Spitzig, R. J. Sober and O. Richmond, The effect of hydrostatic pressure on the deformation behavior of maraging and HY-80 steels and its implication for plasticity theory. Metall. Trans. 7A, 1703-1710 (1976).

[9] I-W. Chen and P. E. Reyes-Morel, Implications of transformation plasticity in $Z_{r} \mathrm{O}_{2}$-containing ceramics: 1 , shear and dilatation effects. J. Am. Ceram. Soc. 69, 181-189 (1986). 
[10] P. E. Reyes-Morel and I-W. Chen, Transformation plasticity of $\mathrm{CeO}_{2}$-stabilized tetragonal zirconia polycrystals: I, stress assistance and autocatalysis. J. Am. Ceram. Soc. 71, 343-353 (1988).

[11] J. W. Hutchinson, Singular behaviour at the end of a tensile crack in a hardening material. J. Mech. Phys. Solids 16, 13-31 (1968).

[12] J. W. Hutchinson, Plastic stress and strain fields at a crack tip. J. Mech. Phys. Solids 16, 337-347 (1968).

[13] J. R. Rice and G. F. Rosengren, Plane strain deformation near a crack tip in a power-law hardening material. J. Mech. Phys. Solids 16, 1-12 (1968).

[14] J. R. Rice, A path independent integral and the approximate analysis of strain concentration by notches and cracks. J. appl. Mech. 35, 379-386 (1968).

[15] J. Pan and C. F. Shih, Plane-strain crack-tip fields for power-law hardening orthotropic materials. Mech. Mater. 5, 299-316 (1986).

[16] J. Pan and C. F. Shih, Plane-stress crack-tip fields for power-law hardening orthotropic materials. Int. J. Fracture 37, 171-195 (1988).

[17] J. Pan, Plane-stress crack tip field for anisotropic perfectly-plastic materials. J. Mech. Phys. Solids 34, 617-635 (1986).

[18] J. Pan, Plane-stress crack-tip fields for perfectly plastic orthotropic materials. Int. J. Fracture 38, 103-122 (1988).

[19] F. Z. Li and J. Pan, Plane-strain crack-tip fields for pressure-sensitive dilatant materials. J. appl. Mech. 57, 40-49 (1990).

[20] F. Z. Li and J. Pan, Plane-stress crack-tip fields for pressure-sensitive dilatant materials. Engng Fracture Mech. 35, $1105-1116$ (1990).

[21] P. Dong and J. Pan, Elastic-plastic analysis of cracks in pressure-sensitive materials. Int. J. Solids Structures 28, 1113-1127 (1991).

[22] P. Dong and J. Pan, Plane-strain mixed-mode near-tip fields in elastic perfectly plastic solids under small-scale yielding conditions. Int. J. Fracture 45, 243-262 (1990).

[23] P. Dong and J. Pan, Plane-stress mixed-mode near-tip fields in elastic perfectly plastic solids. Engng Fracture Mech. 37, 43-57 (1990).

[24] D. C. Drucker and W. Prager, Soil mechanics and plastic analysis or limit design. Q. appl. Math. 10, 157-165 (1952).

[25] J. W. Rudnicki and J. R. Rice, Conditions for the localization of deformation in pressure-sensitive dilatant materials. J. Mech. Phys. Solids 23, 371-394 (1975).

[26] A. Needleman and J. R. Rice, Limits to ductility set by plastic flow localization, in Mechanics of Sheet Metal Forming (Edited by D. P. Koistinen and N.-M. Wang), pp. 237-267. Plenum, New York (1978).

[27] D. R. J. Owen and A. J. Fawkes, Engineering Fracture Mechanics: Numerical Methods and Applications. Pineridge Press, Swansea, U.K. (1983).

[28] M. F. Kanninen and C. H. Popelar, Advanced Fracture Mechancis. Oxford University Press, New York (1985).

[29] K. J. Bathe, Finite Element Procedures in Engineering Mechanics. Prentice-Hall, Englewood Cliffs, NJ (1982).

[30] K. J. Bathe and A. P. Cimento, Some practical procedures for the solution of nonlinear finite element equations. Comput. Meth. appl. Mech. Engng 22, 59-85 (1980).

[31] A. J. Kinloch and R. J. Young, Fracture Behaviour of Polymers. Elsevier Applied Science, London (1983).

[32] J. R. Rice, Elastic-plastic crack growth, in R. Hill 60th Anniversary Volume (Edited by H. G. Hopkins and M. J. Sewell), pp. 539-562. Pergamon Press, Oxford (1982).

[33] Y. C. Gao, Elastic-plastic field of a crack before growing in perfectly plastic medium. Acta Solid Mech. Sinica 1, 69-75 (1980).

[34] S. Nemat-Nasser and M. Obata, On stress field near a stationary crack tip. Mech. Mater. 3, 235-243 (1984).

[35] M. Saka, H. Abe and S. Tanaka, Numerical analysis of blunting of a crack tip in a ductile material under small-scale yielding and mixed mode loading. Comput. Mech. 1, 11-19 (1986).

(Received 2 July 1992) 